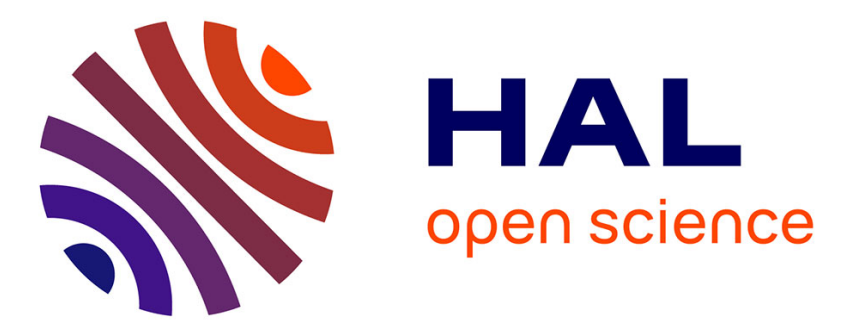

\title{
A low phase noise all cryogenic microwave oscillator based on a superconductor resonator
}

David Chaudy, Olivier Llopis, B. Marcilhac, Y. Lemaître, O. d'Allivy Kelly, J.-M. Hode, J.-M. Lesage

\section{- To cite this version:}

David Chaudy, Olivier Llopis, B. Marcilhac, Y. Lemaître, O. d'Allivy Kelly, et al.. A low phase noise all cryogenic microwave oscillator based on a superconductor resonator. IEEE Transactions on Ultrasonics, Ferroelectrics and Frequency Control, 2020, 67 (12), pp.2750 - 2756. 10.1109/TUFFC.2020.3023198 . hal-02948581

\section{HAL Id: hal-02948581 \\ https://hal.laas.fr/hal-02948581}

Submitted on 24 Sep 2020

HAL is a multi-disciplinary open access archive for the deposit and dissemination of scientific research documents, whether they are published or not. The documents may come from teaching and research institutions in France or abroad, or from public or private research centers.
L'archive ouverte pluridisciplinaire HAL, est destinée au dépôt et à la diffusion de documents scientifiques de niveau recherche, publiés ou non, émanant des établissements d'enseignement et de recherche français ou étrangers, des laboratoires publics ou privés. 


\title{
A low phase noise all cryogenic microwave oscillator based on a superconductor resonator
}

\author{
D. Chaudy, O. Llopis, B. Marcilhac, Y. Lemaître, O. d'Allivy Kelly, J.-M. Hode, J.-M. Lesage
}

\begin{abstract}
A $1 \mathrm{GHz}$ full cryogenic oscillator is presented. The oscillator is based on a planar superconductor resonator featuring a loaded $Q$ factor of 200000 at low microwave input power (unloaded $Q$ of 400000 ) and on amplifying parts realized with SiGe bipolar transistors. The circuit is designed with an harmonic balance software and realized on alumina substrate. A nonlinear model is extracted at low temperature both for the transistor and the resonator. This double nonlinearity increases the difficulty of the oscillator design and implies a strategy to limit the power inside the resonator. The vibrations of the cryogenerator are also a serious issue to get high performance. Finally, the oscillator features a phase noise of $-112 \mathrm{dBc} / \mathrm{Hz}$ at $100 \mathrm{~Hz}$ offset frequency and a phase noise floor of $-170 \mathrm{dBc} / \mathrm{Hz}(100 \mathrm{kHz}$ offset $)$ at a temperature of $65 \mathrm{~K}$.
\end{abstract}

Index Terms - microwave oscillators, phase noise, nonlinear modeling, high-temperature superconductors, superconducting resonator, cryogenic electronics

\section{INTRODUCTION}

Improving the phase noise of microwave sources is a constant challenge, which has an impact on different applications fields such as high performance radars and atomic clocks. In an oscillator loop, the phase noise is directly related to the amplifier open loop phase noise and is inversely proportional to the square of the resonator quality factor Q. Various technologies allows to get an high $\mathrm{Q}$ resonance at high frequencies. Piezoelectric resonator is the standard technology in the radio frequency $(\mathrm{RF})$ range, i.e. below $1 \mathrm{GHz}$. Today, most commercially available microwave sources are based on the frequency multiplication of RF quartz sources. This technique however increases the phase noise floor far from the carrier and high $\mathrm{Q}$ resonators in the microwave range (above $1 \mathrm{GHz}$ ) are of great interest to get a better performance at frequency offsets in the range of $10 \mathrm{kHz}$.

Manuscript received on...

The work is supported by the French MoD, Direction Générale de l'Armement (DGA).

D. Chaudy, O. Llopis are with LAAS-CNRS, Université de Toulouse, CNRS, UPS, 7 av. Du Colonel Roche, 31031 Toulouse, France. (e-mail : llopis@laas.fr).

B. Marcilhac, Y. Lemaître, O. d'Allivy Kelly are with Unité Mixte de Physique, CNRS, Thales, Paris-Sud Université Paris-Saclay, 91767, Palaiseau, France

J.-M. Hode is with Thales Defense Mission Systems, av. Marcel Dassault, 33700, Mérignac, France.

J.-M. Lesage is with DGA, French MoD, 35170 Bruz, France.
Dielectric resonator technology, which uses low loss dielectric ceramic materials, is a good choice for microwave local oscillators [1]. However, dielectric resonators Q factor stays in the range of a few $10^{4}$ at $10 \mathrm{GHz}$ and has not improved recently. An alternative to ceramic materials is single crystal sapphire resonators, with $\mathrm{Q}$ in the range of $10^{5}$. Impressive results have been obtained with this technology, both at ambient [2] and low temperature $[3,4]$. However, sapphire is a low permittivity material and this type of resonator needs to be embedded in large metallic cavity, which increases the size of the system. Besides the problem of size, sapphire thermal coefficient is quite large at ambient temperature and $300 \mathrm{~K}$ sapphire oscillators feature poor long-term stability (this is not the case of the cryogenic sapphire oscillator, which works close to liquid helium temperature).

The use of optical resonators or optical delay lines is another technique to reach ultra-high $\mathrm{Q}$ factors and high quality oscillation in the microwave range [5,6]. However, once again the systems are very often bulky and complex because of the use of laser, modulator, photodiode and, sometimes, electronic stabilization loops. Some compact optoelectronic sources based on whispering gallery mode resonators have been proposed [6] but their performance is still not at the level of sapphire oscillators or long delay line optoelectronic oscillators.

In any case, $\mathrm{Q}$ factor is directly related to the losses in the resonator: it is proportional to the ratio of the stored energy to the energy loss per cycle (this is why sapphire material, with its extremely low loss tangent or optical resonator or optical fiber are so interesting). To get high performance in a small volume, it is interesting to combine the low losses properties of dielectric materials at low temperature with the exceptional properties of superconductors. If the losses in the conducting parts are negligible, it becomes possible to get ultra high $\mathrm{Q}$ with microstrip technology. With this approach, high quality planar microwave resonators can be realized with high temperature superconductors (HTS) thin films. The superconductor surface resistance is dependent on the operating frequency and on the temperature. It degrades with $\mathrm{f}^{2}$ when the frequency increases and this explains why HTS technology performs better in the low microwave range. Contrarily to the first projects on hybrid superconductor oscillators in the 1990s [7,8], we have chosen to work at $1 \mathrm{GHz}$, which allows to reach unloaded quality factors higher than 200000 for this type of resonator at $77 \mathrm{~K}$ 
$[9,10]$. Besides, $1 \mathrm{GHz}$ is a popular frequency to generate frequency combs used to stabilize synthesizers in the whole microwave range.

However, the resonator is not the only problem in the design of a high performance frequency source. The amplifier sustaining the oscillation should feature a phase noise as low as possible. It must be optimized by choosing a low $1 / \mathrm{f}$ noise transistor and the reduction of the conversion process of $1 / f$ noise into phase noise with a proper choice of HF and LF loads [11]. We focus in this paper on SiGe bipolar transistors which combine low 1/f noise and high gain at low temperature [12, 13].

Finally, the remaining problem with the circuit design is that any change of a device or any device adjustment inside the cryostat requires a time consuming process of cooling up and down the circuit. Therefore, a reliable nonlinear model at low temperature must be extracted, both for the resonator and the transistor. This model is presented in this paper. It has allowed the design and optimization of different types of all cryogenic circuits: a low phase noise amplifier and two loop oscillators, with and without output buffer. Phase noise results obtained on these different circuits are presented and discussed.

\section{ACTIVE DEVICES SELECTION AND MODELLING}

The devices selection process has been presented in the reference [14]. It is based on the measurement of the transistor open loop (or residual) phase noise at the frequency of interest and on the measurement of its gain properties at low temperature ( $\mathrm{S}$ parameters). To this purpose, a residual phase noise measurement bench at $1 \mathrm{GHz}$ has been set-up. It uses a low phase noise $1 \mathrm{GHz}$ source (NEL multiplied OCXO) and two low 1/f noise RF mixers (Mini-circuits) to perform a cross correlation analysis.

Four different SiGe transistors have been measured, both at ambient and low temperature $(80 \mathrm{~K})$. These devices feature good properties at $80 \mathrm{~K}$, with a $50 \Omega$ gain $\left(\mathrm{S}_{21}\right)$ which is increased by $2 \mathrm{~dB}$ in average and a $1 / \mathrm{f}$ phase noise component which degrades for some devices by $10 \mathrm{~dB}$, but only by $2 \mathrm{~dB}$ for the best devices. Based on these measurements, a SiGe transistor has been selected for a complete nonlinear modeling at $80 \mathrm{~K}$ : a BFP650 device from Infineon.

The model is performed at $80 \mathrm{~K}$ because the use of liquid nitrogen allows a faster cooling of the devices. The final circuit temperature being between $60 \mathrm{~K}$ and $70 \mathrm{~K}$, a check of the model validity at $60 \mathrm{~K}$ is performed on the simplest circuit, the amplifier. The transistor model is developed on Keysight ADS software. It is based on the Gummel-Poon model [15] given by the device manufacturer which has been adjusted to the measured low temperature data. The $\mathrm{I}_{\mathrm{ce}}\left(\mathrm{V}_{\mathrm{ce}}\right)$ curves and the Gummel plot $\left(\mathrm{I}_{\mathrm{c}}\right.$ and $\mathrm{I}_{\mathrm{b}}$ versus $\mathrm{V}_{\mathrm{be}}$ ) at $80 \mathrm{~K}$ allow to fit the SPICE model parameters and the external parasitic elements (series inductors and parallel capacitors) are used to adjust the device $\mathrm{S}$ parameters. These data, measured and modeled, are depicted in Figure 1 and 2.

A check of the model is performed through the measurement of the nonlinear behavior when the device is driven into compression. As depicted in Fig. 3, the general behavior of the model is in good agreement with the measured data, even if some differences can be observed on the second harmonic.

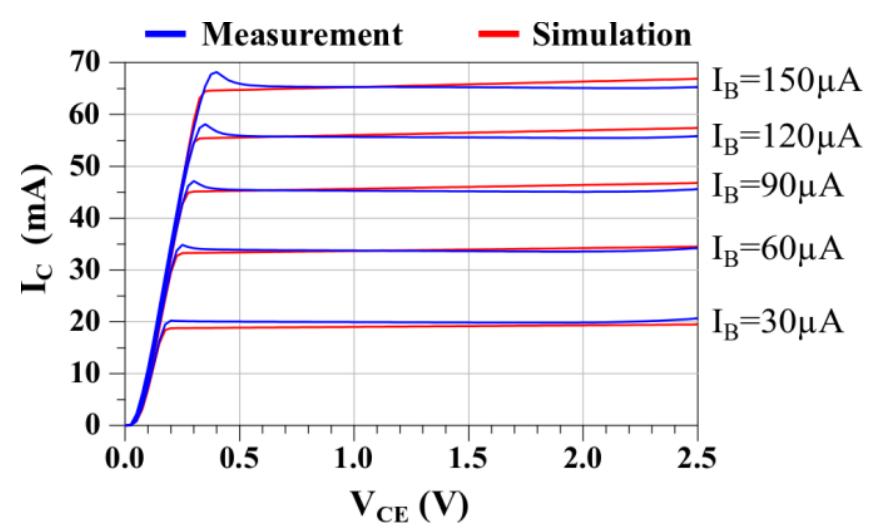

Figure 1: I(V) plot for the BFP650 device at $80 \mathrm{~K}$ (simulated and measured)
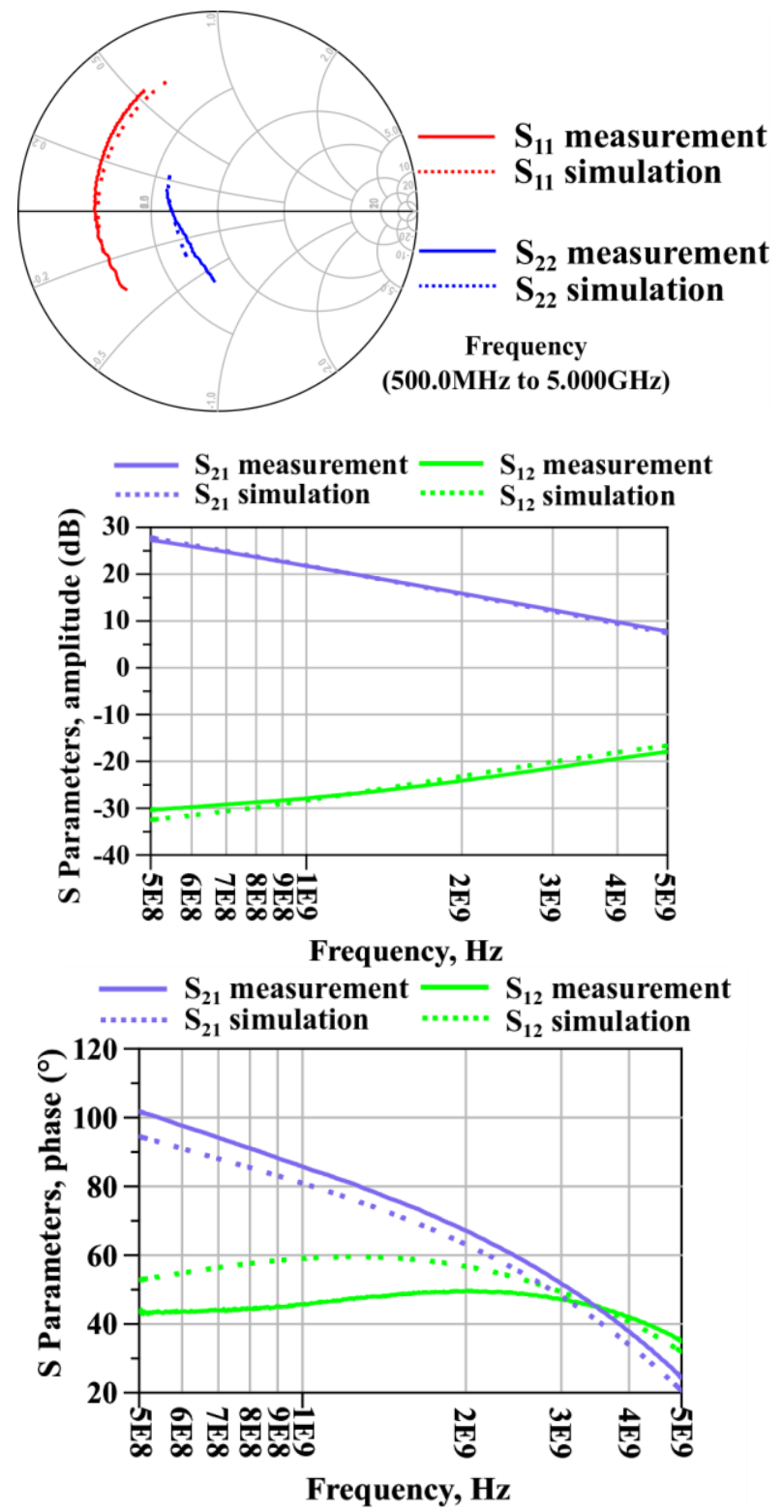

Figure 2: S parameters of BFP650 device at $80 \mathrm{~K}$ (simulated and measured) 


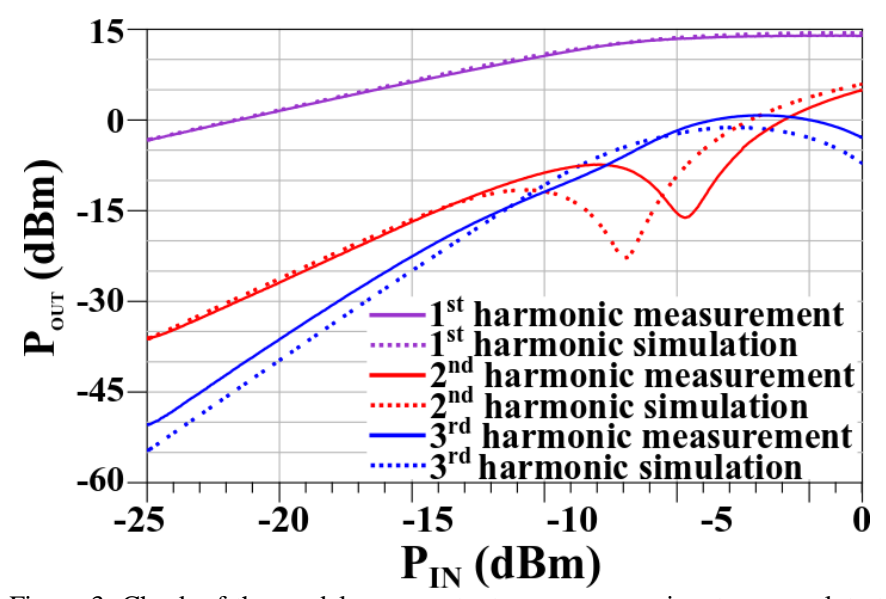

Figure 3: Check of the model on an output power versus input power plot at $1 \mathrm{GHz}$ and $80 \mathrm{~K}$, for the first, second and third harmonics. $1 \mathrm{~dB}$ compression is reached for approximately $-7 \mathrm{dBm}$ input power.

Finally, the last step is to add the noise sources to the model. We use a low impedance bias for the device [11] in order to minimize the impact of the base-emitter current noise source to the phase noise. With this configuration, the main contribution to the phase noise comes from a voltage fluctuation on the base. Therefore, a 1/f noise source is added on this electrode in the model in order to fit the measured residual phase noise of the device at $1 \mathrm{~dB}$ compression point (Figure 4). No attempt has been made to develop a model with cyclostationary noise sources. We make the assumption that the device will work in the final circuit in similar conditions than for this $1 \mathrm{GHz}$ residual phase noise measurement.

The transistor is not the only device used in the cryogenic oscillator. The oscillation loop involves various passive devices, which have been checked versus their low temperature behavior (resistors, capacitors, inductors, alumina substrate). No significant change has been observed on these devices at $1 \mathrm{GHz}$ and $80 \mathrm{~K}$, compared to their values at $300 \mathrm{~K}$. The last device which is used in the loop, in order to tune the oscillation frequency or, eventually, to start the oscillation, is a phase shifter realized with a series mounted varactor diode. Again, very little variation is observed on this device while cooling it down to $80 \mathrm{~K}$, as shown in Table 1 . We have also performed residual phase noise measurements on this varactor diode, mounted in series, and no significant noise contribution has been observed with this device.

\begin{tabular}{|l|c|c|c|}
\hline BB837 bias voltage & $0 \mathrm{~V}$ & $10 \mathrm{~V}$ & $20 \mathrm{~V}$ \\
\hline$\left|\mathrm{S}_{21}\right| @ 1 \mathrm{GHz} 300 \mathrm{~K}$ & $-0.3 \mathrm{~dB}$ & $-4.9 \mathrm{~dB}$ & $-9.0 \mathrm{~dB}$ \\
\hline$\left|\mathrm{S}_{21}\right| @ 1 \mathrm{GHz} 80 \mathrm{~K}$ & $-0.2 \mathrm{~dB}$ & $-5.1 \mathrm{~dB}$ & $-9.1 \mathrm{~dB}$ \\
\hline$\Phi_{\mathrm{S} 21} @ 1 \mathrm{GHz} 300 \mathrm{~K}$ & $3^{\circ}$ & $51^{\circ}$ & $65^{\circ}$ \\
\hline$\Phi_{\mathrm{S} 21} @ 1 \mathrm{GHz} 80 \mathrm{~K}$ & $5^{\circ}$ & $52^{\circ}$ & $66^{\circ}$ \\
\hline
\end{tabular}

Table 1: $\mathrm{S}_{21}$ parameter at $300 \mathrm{~K}$ and $80 \mathrm{~K}$ for one of the varactor diodes measured. No significant variation is observed on this device with cooling.

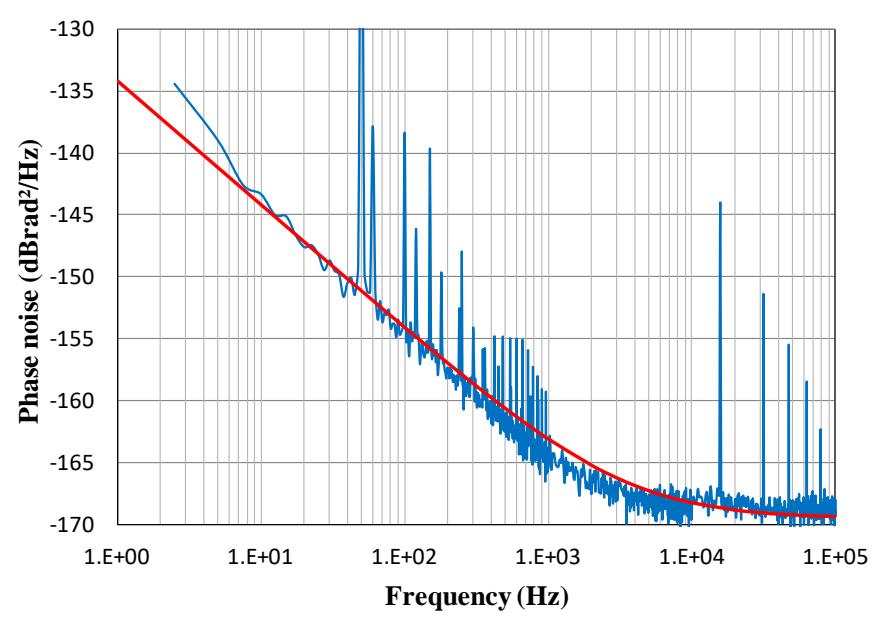

Figure 4: Simulated (red) and measured BFP650 $1 \mathrm{GHz}$ phase noise at $80 \mathrm{~K}$. The device is loaded onto $50 \Omega$ at RF, low impedance on the base at DC and the $\mathrm{RF}$ input power corresponds to $1 \mathrm{~dB}$ power compression. The measurement is performed in a liquid nitrogen dewar (transistor maintained above the liquid nitrogen level and cooled thanks to a brass cold finger).

\section{LOW PHASE NOISE AMPLIFIER DESIGN}

The low phase noise amplifier should be able to sustain an oscillation on a moderately coupled resonator, i.e. with $6 \mathrm{~dB}$ losses. The BFP650 device features an $S_{21}$ of more than $20 \mathrm{~dB}$ at the frequency of interest $(1 \mathrm{GHz})$, which is too high for an optimized oscillator $[11,16]$. Therefore, the gain of the transistor has to be reduced and this should go with an improvement of its phase noise and stability. To this purpose, two different feedback loops have been added: a series feedback on the emitter and a parallel feedback between collector and emitter. Indeed, a series feedback with a few ohms resistor reduces the conversion of the transistor low frequency noise [17]. Also, to minimize this effect, a compromise has to be found on the transistor bias point: a high current bias point increases the device 1/f noise on the base emitter access but also reduces the conversion of this noise into phase noise [18]. The optimization of all these parameters can only be performed using a non-linear model of the amplifier. Therefore, gain, stability and phase noise have been optimized using the transistor model described in paragraph II.

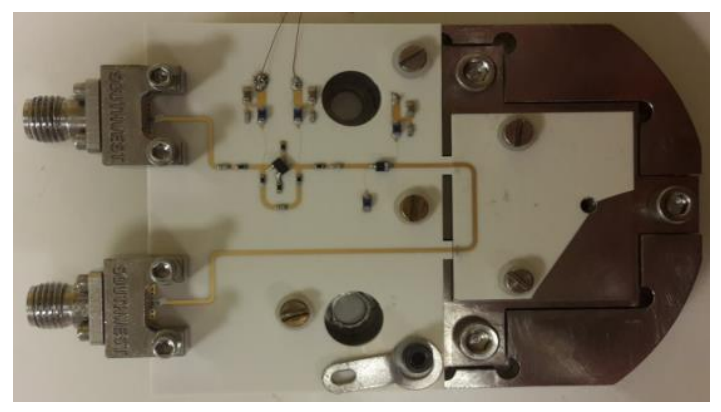

Figure 5: $1 \mathrm{GHz}$ amplifier circuit designed for the test in open loop mode.

The circuit designed is depicted in Figure 5. It includes the transistor bias circuit, the two feedbacks (series and parallel) and a varactor diode in series with the transistor to control the 
phase. For these measurements in open loop configuration (amplifier only), the resonator is replaced by a U-shaped microstrip line (right part on the photograph).

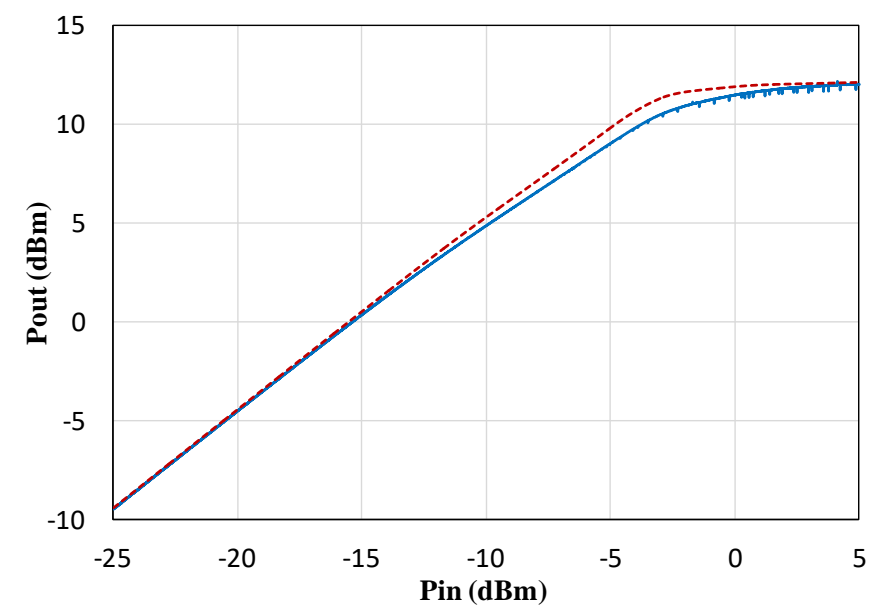

Figure 6: $1 \mathrm{GHz}$ amplifier output power versus input power at $80 \mathrm{~K}$, measured (blue) and simulated data (red dotted line)

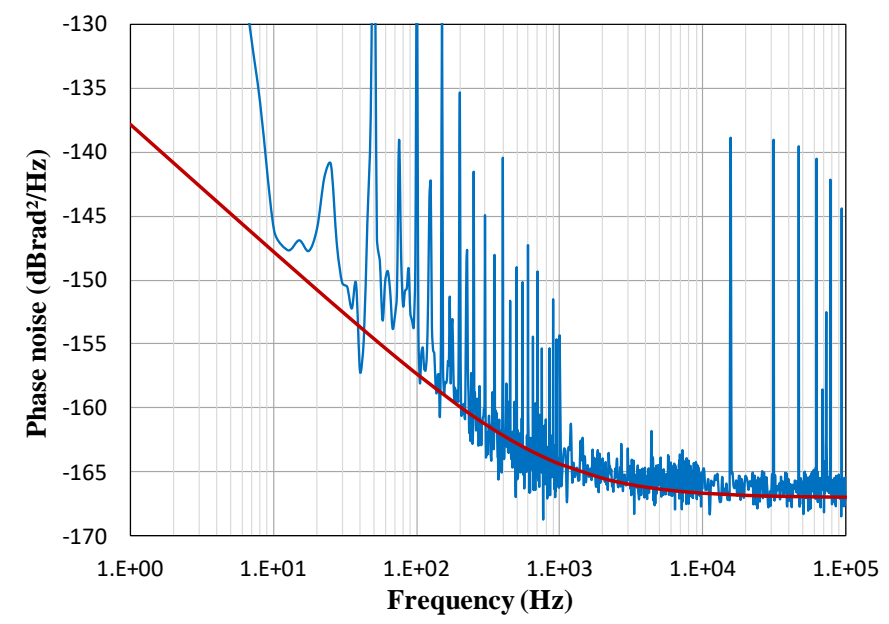

Figure 7: Amplifier residual phase noise measured at $85 \mathrm{~K}$ and $1 \mathrm{GHz}$, and simulated using ADS (red curve). The RF input power is $-8 \mathrm{dBm}$. Compared to the transistor performance (Figure 4), the 1/f phase noise is $4 \mathrm{~dB}$ lower.

The measured and simulated amplifier gain and residual phase noise performances are depicted respectively in Figure 6 and 7. These measurements have been performed with the circuit maintained in a cryocooler to avoid temperature fluctuations and to try to reach low enough temperatures for further experiments with superconducting cavities. However, the temperature stayed at a minimum of $85 \mathrm{~K}$ because of the amount of heat brought back by the two coaxial cables.

When the residual phase noise results (Fig. 7) are compared to the one of the transistor alone (Figure 4), it is obvious that the phase noise of the amplifier is reduced in the 1/f noise region, even if the cryostat vibrations did not allow a clear measurement at low offset frequencies. The $1 / \mathrm{f}$ phase noise corner frequency computed from the simulated data is $1 \mathrm{kHz}$ for the amplifier. This cryogenic amplifier is thus a particularly interesting circuit for cryogenic oscillator applications.

\section{HIGH TC SUPERCONDUCTOR RESONATOR MODELLING}

The resonator is the other important device of the loop. It should feature an intrinsic quality factor $\mathrm{Q}_{\mathrm{o}}$ as high as possible and a coupling factor tuned to get $6 \mathrm{~dB}$ to $10 \mathrm{~dB}$ losses in transmission, in order to keep an high enough loaded quality factor $\mathrm{Q}_{\mathrm{L}}\left(Q_{L}=\frac{Q_{0}}{2}\right.$ with $6 \mathrm{~dB}$ losses $)$ without degrading too much the signal to noise ratio in the loop [1,11].

The research at UMR CNRS-Thales on HTS has led to the development of planar resonators with $\mathrm{Q}_{0}$ higher than 200000 at $1 \mathrm{GHz}$ using Ceraco Ceramic Coating $\mathrm{GmbH}$ technology based on $\mathrm{Y}_{1} \mathrm{Ba}_{2} \mathrm{Cu}_{3} \mathrm{O}_{7-\mathrm{x}}$ thin films on $\mathrm{MgO}$ substrate $[9,10,19]$. The resonators are also optimized for their power handling capabilities for a given surface.

A simple application of Leeson's equation [20] using the measured amplifier phase noise $\left(-165 \mathrm{~dB}_{\mathrm{rad}^{2}}^{2} / \mathrm{Hz}\right.$ at $\left.1 \mathrm{kHz}\right)$ and a $\mathrm{Q}_{\mathrm{L}}$ value of 100000 lead to a phase noise level of $-154 \mathrm{~dB}_{\mathrm{c}} / \mathrm{Hz}$ at $1 \mathrm{kHz}$ for the $1 \mathrm{GHz}$ cryogenic oscillator.

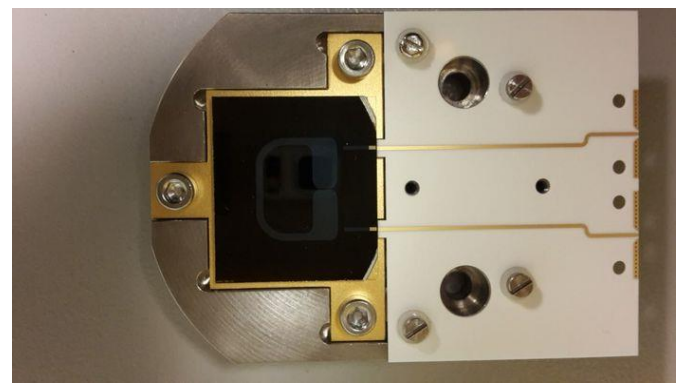

Figure 8: Superconducting resonator: $\mathrm{YBCO}$ film deposited on a $\mathrm{MgO}$ substrate by Ceraco $\mathrm{GmbH}$, on the left side of the photograph. The upper part of the resonator (not shown here) is made of copper. On the rigth side, the oscillator circuit is replaced by two microstrip lines for the resonator characterization.

\begin{tabular}{|c|c|c|c|}
\hline $\begin{array}{c}\text { Input Power } \\
\mathbf{d B m}\end{array}$ & $\mathbf{S}_{\mathbf{2 1} \text { Max }} \mathbf{d B}$ & Loaded Q & Unloaded Q \\
\hline-20 & -6.0 & 200000 & 420000 \\
\hline-15 & -6.1 & 195000 & 405000 \\
\hline-10 & -6.3 & 193000 & 390000 \\
\hline-5 & -6.6 & 180000 & 350000 \\
\hline 0 & -8.0 & 150000 & 260000 \\
\hline 5 & -11.1 & 110000 & 150000 \\
\hline 10 & -14.9 & 69000 & 84000 \\
\hline
\end{tabular}

Table 2: $\mathrm{Q}$ factor and transmission losses degradation with RF power at $60 \mathrm{~K}$ and $1 \mathrm{GHz}$, in the last generation of HTS resonators designed.

In this study, the technology of the resonators has been optimized to reach unloaded Q factors higher than 400000 at $1 \mathrm{GHz}$ and $60 \mathrm{~K}$, as shown in Table 2, particularly through a redefinition of the copper housing dimensions. However, the superconductor resonator is not a purely passive device. With such quality factors, the power enhancement factor inside the cavity is huge and the surface currents on the superconductor increase rapidly. This behavior induces Joule effect resulting in the separation of the electrons pairs and vortex motion, increasing the normal current component which reduces the quality factor [21-23]. This $\mathrm{Q}$ factor degradation at high input power can be considered, from a circuit point of view, as a nonlinearity. The $\mathrm{Q}$ factor is not the only problem in this process: the transmission losses through the resonator increase 
when this phenomenon occurs and this limit the oscillation amplitude just like the saturation effect of the amplifier (see Table 2). Moreover, the resonator frequency is also slightly shifted towards lower frequencies because of the change is the superconductor skin effect.

In order to take into account this behavior in the simulations, an RLC circuit model with two nonlinear elements has been developed [8]. The model is depicted in Figure 9 and the nonlinear elements are the intrinsic resistor $\mathrm{R}$ and inductor $\mathrm{L}$ which are dependent on the instantaneous current $I$ in the resonator.

$$
\begin{aligned}
& \mathrm{R}=\mathrm{R}_{0}\left(1+\alpha I^{2}+\beta I^{4}\right) \\
& \mathrm{L}=\mathrm{L}_{0}\left(1+\gamma I^{2}\right)
\end{aligned}
$$

This model is able to describe the measured resonator frequency response when the empirical parameters $\alpha, \beta$ and $\gamma$ are correctly tuned, as depicted in Figure 10 for the quasi linear conditions (input power $-20 \mathrm{dBm}$ ) and for the saturation conditions (input power $0 \mathrm{dBm}$ ).

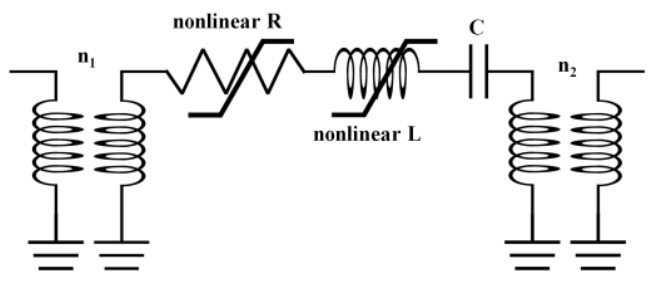

Figure 9: Nonlinear HTS-resonator model

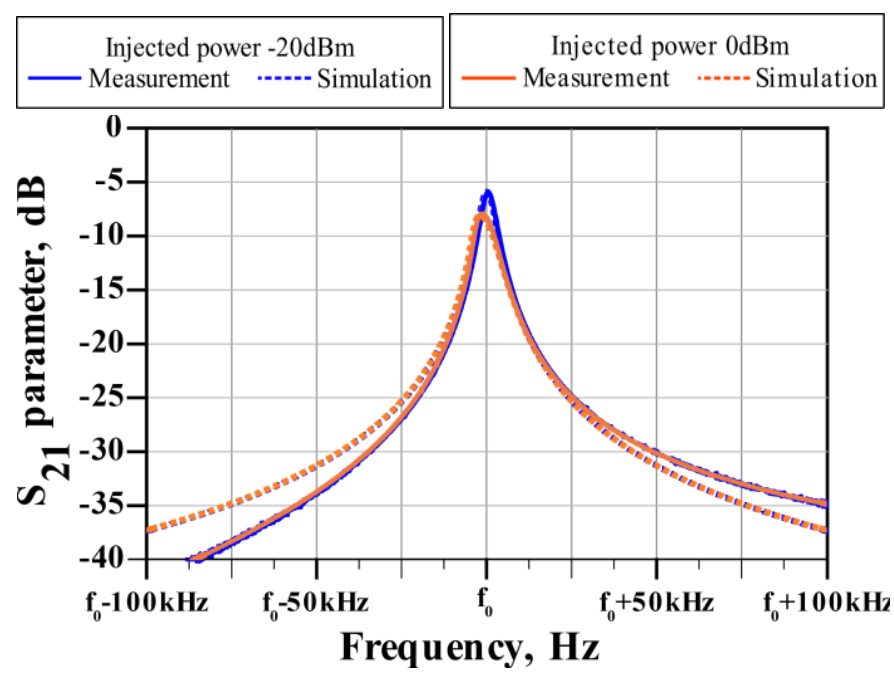

Figure 10: Transmition parameters of an HTS-Resonator at $60 \mathrm{~K}\left(\mathrm{f}_{0} \sim 1 \mathrm{GHz}\right)$

\section{CRYOGENIC OSCILLATOR DESIGN AND MEASUREMENT}

The amplifier model and the resonator model being validated, the oscillator design can start. Compared to classical microwave oscillator design, the difficulty here is to take into account the two main nonlinearities of the circuit: the transistor and the resonator. Because the compression of the resonator results in a strong degradation of its $\mathrm{Q}$ factor, the oscillating power should be controlled by the amplifier. A careful management of the power around the oscillating loop has thus been realized thanks to the simulation. The transistor bias point is chosen to deliver a relatively low output power and the cascaded power splitters and attenuators helps in maintaining the resonator input power at about $-5 \mathrm{dBm}$. Because of this low oscillation power and in order to isolate the oscillation from the outside world, a buffer amplifier is added to the circuit. With this output buffer, the circuit delivers $9 \mathrm{dBm}$ RF power at $1 \mathrm{GHz}$. Compared to our first cryoelectronic oscillator design [10], the circuit is now much more complex and includes not only this output buffer but also all the bias circuits for the two amplifiers, as depicted in Figure 11 and 12.

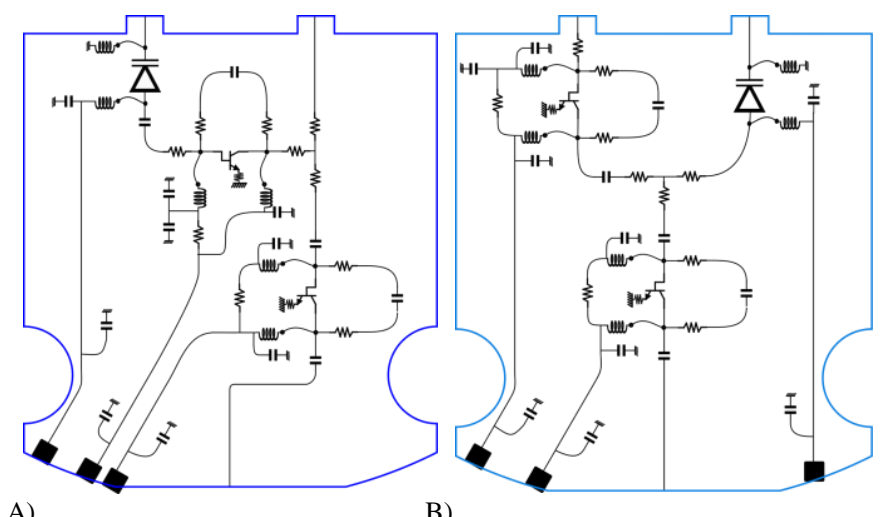

Figure 11: Schematics of the two circuits realized, including a varactor diode on the superconducting resonator output (left: A) or input (rigth: B), and a buffer amplifier on the signal output path

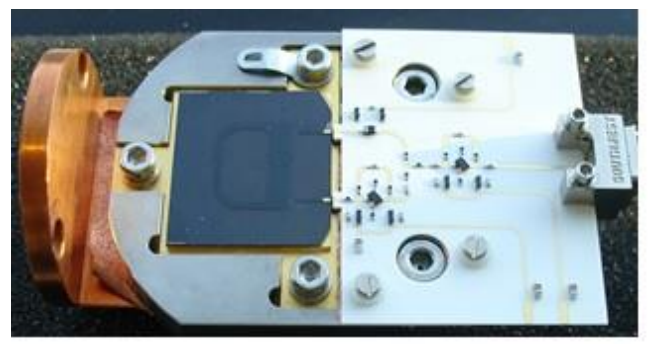

Figure 12: Full cryogenic HTS oscillator with output buffer (B type).

Superconductor resonator on the left and active circuit on the rigth side.

The circuit simulations, including the phase noise, have been performed on ADS using the harmonic balance approach. Then the system has been included in a cryostat, based on a pulse tube cryocooler LPT9310 from Thales Cryogenics, and has been measured at $65 \mathrm{~K}$. The circuit is maintained on the cryostat cold finger and thermally isolated from the output using an RF thermal bridge realized with wire bondings. Because of the problem of power management for the HTS resonator, the loop gain was too low in the first experiments and some loop resistors included to attenuate the signal before the resonator have then been removed.

Figure 13 depicts the $1 \mathrm{GHz}$ oscillator phase noise measured with a Rohde \& Schwarz FSWP26 signal analyzer which is compared to the simulated phase noise obtained from ADS. These data correspond to the case of circuit B (Figure 11) but a similar level of phase noise in the $1 / \mathrm{f}$ noise region has been measured on circuit A. To perform this measurement in optimized conditions versus mechanical vibrations, the 
cryogenerator was cut-off during the measurement and the vacuum pump was connected through a flexible hose. Under these conditions, the measurement acquisition needs to be fast enough and the correlation number is limited to 20 on the lower frequency band $(30 \mathrm{~Hz}$ to $300 \mathrm{~Hz}$ ) and then increases progressively at higher frequency offsets. Also, the spur removal option was activated on the FSWP signal analyzer. Finally, the varactor (phase shifter) has been short-circuited to be sure that no additional noise could be induced by this device.

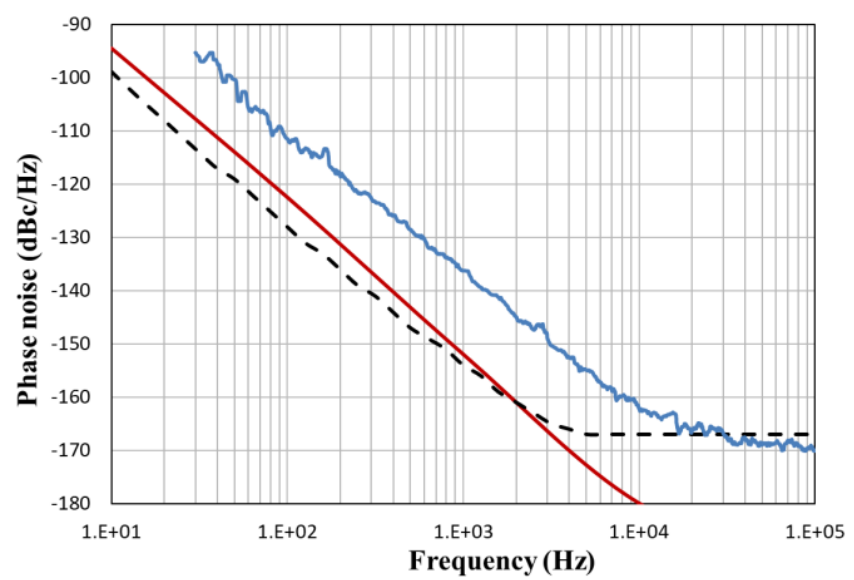

Figure 13: $1 \mathrm{GHz}$ full cryogenic HTS oscillator with output buffer at $65 \mathrm{~K}$. Phase noise measurements results (blue curve) compared to the simulation (black dashed line). The estimated measurement system noise floor, taking into account the correlation number, is also shown (red line).

Contrarily to the amplifier case, we can see here a strong difference between the simulated and the measured phase noise, of about $17 \mathrm{~dB}$ in the $1 / \mathrm{f}$ noise region of the spectrum. The measurement system noise floor being lower than the measured phase noise, we suppose a change in the circuit operating conditions compared to the simulation, or an additional noise source not taken into account in the simulation. To start the oscillation, the loop power has been increased and a compression of the resonator higher than the one simulated could partly explain this performance degradation. However, the $\mathrm{Q}$ factor should be divided by a factor 7 to explain such a behavior, which is much too large compare to what has been observed on the resonator alone. Therefore, an additional noise source in the oscillating loop is suspected to degrade the oscillator performance. This noise source could come from the HTS resonator, as it is the only different device in the circuit compared to the amplifier case. However, contact problems with the soldered devices cannot be completely dismissed. Indeed, a non perfect ohmic contact may generate 1/f noise when submitted to a DC current [24].

Anyway, even with this additional noise, the cryoelectronic oscillator phase noise performances are still very interesting: $-112 \mathrm{dBc} / \mathrm{Hz} @ 100 \mathrm{~Hz},-137 \mathrm{dBc} / \mathrm{Hz} @ 1 \mathrm{kHz}$ and a phase noise floor at $-170 \mathrm{dBc} / \mathrm{Hz} @ 100 \mathrm{kHz}$. This measured phase noise spectrum is similar to the one of the best SAW oscillators or to the best $100 \mathrm{MHz}$ OCXO multiplied to $1 \mathrm{GHz}$. As an example, a Rakon $1 \mathrm{GHz}$ multiplied by two $500 \mathrm{MHz}$ SAW oscillator feature a phase noise level of $-105 \mathrm{dBc} / \mathrm{Hz} @ 100 \mathrm{~Hz}$, $-135 \mathrm{dBc} / \mathrm{Hz} @ 1 \mathrm{kHz}$ and $-172 \mathrm{dBc} / \mathrm{Hz} @ 100 \mathrm{kHz}$. A NEL
OCXO multiplied to $1 \mathrm{GHz}$ feature a phase noise of $-115 \mathrm{dBc} / \mathrm{Hz} @ 100 \mathrm{~Hz},-140 \mathrm{dBc} / \mathrm{Hz} @ 1 \mathrm{kHz}$ and -160 $\mathrm{dBc} / \mathrm{Hz} @ 100 \mathrm{kHz}$ (noise floor). Contrarily to these two technologies, which have been constantly optimized during the last 30 or 50 years, the oscillator presented here is the first one of his kind. It features therefore the potential for strong optimization if the noise contributors can be all understood.

\section{CONCLUSION}

A $1 \mathrm{GHz}$ full cryogenic oscillator based on a high Tc superconductor resonator has been presented, together with the methodology for its design: transistor choice, transistor modeling, amplifier design, amplifier measurement, resonator characterization, resonator modeling and, finally, oscillator design and characterization. The amplifier performance at low temperature, with a phase noise floor at $-170 \mathrm{dBc} / \mathrm{Hz}$ and a $1 / \mathrm{f}$ corner at about $1 \mathrm{kHz}$, is particularly interesting for cryogenic applications sensitive to phase fluctuations, including other types of oscillators. The HTS resonator based oscillator phase noise performance is not at the level expected at the beginning of this study and predicted by the model. However, it can be compared to the performance of the best quartz based sources and should be largely improved in the future if all the noise sources can be clearly identified in this circuit and if all the vibration issues can be solved.

\section{REFERENCES}

[1] J. Everard, K. Theodoropoulos, "Ultra low phase noise ceramic based dielectric resonator oscillators", Proc. of the IEEE-International Frequency Control Symposium, Miami, June 2006.

[2] E. Ivanov, M. Tobar, "Low phase noise microwave oscillators with interferometric signal processing", IEEE Trans. on Microwave Theory and Tech., vol 54, nº 8, August 2006, pp. 3284-3294.

[3] C. R. Locke, E. N. Ivanov, J. G. Hartnett, P. L. Stanwix, and M. E. Tobar, "Design techniques and noise properties of ultrastable cryogenically cooled sapphire-dielectric resonator oscillators", Rev. of Scientific Instrum., 79, 051301, 2008

[4] S. Grop, P.-Y. Bourgeois, R. Boudot, Y. Kersale, E. Rubiola and V. Giordano,"10 GHz cryocooled sapphire oscillator with extremely low phase noise," Electron. Letters, vol. 46, no. 6, March 2010, pp. 420-422.

[5] D. Eliyahu, D. Seidel, L. Maleki, "RF Amplitude and Phase-Noise Reduction of an Optical Link and an Opto-Electronic Oscillator", IEEE Trans. on MTT, Vol. 56, $\mathrm{N}^{\circ} 2$ 2, Feb. 2008, pp. 449-456.

[6] L. Maleki, "The optoelectronic oscillator", Nature Photonics (Technology focus), vol. 5, Dec. 2011, pp. 728-730.

[7] N. J. Rohrer, G. J. Valco, K. B. Bhasin, "Hybrid High Temperature Superconductor/GaAs $10 \mathrm{GHz}$ Microwave Oscillator: Temperature and Bias Effects", IEEE Trans. on MTT, vol. 41, n 11, Nov. 1993, pp. 18651871.

[8] G. Borghs, J. Deboeck, I. Francois, D. Chambonnet, C. Belouet, et al., "A $12 \mathrm{GHz}$ oscillator based on a GaAs HEMT integrated to a HTS resonator" Journal de Physique IV Colloque, 1994, 04 (C6), pp. C6-189-C6-194.

[9] J.-C. Mage, B. Marcilhac, M. Poulain, Y. Lemaître, J. Kermorvant, J.M. Lesage, "Low noise oscillator based on 2D superconducting resonator", Proc. of the EFTF \& IEEE-IFCS joint conference, San Francisco, May 2011.

[10] D. Chaudy, O. Llopis, B. Marcilhac, Y. Lemaître, O. D'Allivy Kelly, J.M. Hode, "Low phase noise cryogenic amplifiers and oscillators based on superconducting resonators", Proc. of the IEEE Int. Frequency Control Symposium, Lake Tahoe, California, May 2018.

[11] O. Llopis, G. Cibiel, "Phase noise metrology and modelling of microwave transistors - Applications to the design of the state of the art dielectric resonator," Proc. SPIE Vol. 5113, Noise in Devices and Circuits, Inv. presentation at Int. Symp. on Fluctuations and Noise, Santa Fe, June 2003. 
[12] S. Gribaldo, PhD thesis (in french), Toulouse University, 2008.

[13] S. Weinreb, J. C. Bardin, H. Mani, "Design of Cryogenic SiGe Low-Noise Amplifiers", IEEE Trans. on MTT, Vol. 55, N 11, nov. 2007, pp. 23062312.

[14] D. Chaudy, O. Llopis, B. Marcilhac, Y. Lemaître, O. d'Allivy Kelly, J.M. Hode "Active devices choice and design of an all cryogenic superconductor resonator oscillator", IEEE Int. Frequency Control Symposium \& EFTF joint Meeting, Besançon, July 2017.

[15] G. Massobrio and P. Antognetti, "Semiconductor Device Modelling with SPICE", McGraw-Hill Ed., 1998.

[16] M. Régis, O. Llopis, J. Graffeuil, "Nonlinear modeling and design of bipolar transistors ultra low phase noise dielectric resonator oscillators" IEEE trans. on Microwave Theory Tech., Vol 46, $\mathrm{N}^{\circ}$ 10, Oct. 1998, pp. 1589-1593.

[17] F. L. Walls, E. S. Ferre-Pikal, S. R. Hefferts, "Origin of 1/f PM and AM noise in bipolar junction transistor amplifiers," IEEE Trans. Ultrason., Ferroelect., Freq. Contr., vol. 44, no. 2, pp. 326-334, Mar. 1997.

[18] O. Llopis, J. M. Dienot, J. Verdier, R. Plana, M. Gayral, and J. Graffeuil, "Analytical investigation of frequency sensitivity in microwave oscillators: Application to the computation of phase noise in dielectric resonator oscillators," Ann. Telecom., vol. 51, n 3-4, pp. 121-129, 1996.

[19] J. Kermorvant, $\mathrm{PhD}$ thesis (in french), Ecole Polytechnique, 2010.

[20] D. B. Leeson, "A simple model of feedback oscillator noise spectrum", Proc. of the IEEE, vol 54, n 2, Feb. 1966, pp. 329-330.

[21] J. Kermorvant, J.-C. Mage, B. Marcilhac, Y. Lemaître, J.-F. Bobo, C. Jacominus van der Beek, "Microwave heating-induced static magnetic flux penetration in $\mathrm{YBa} 2 \mathrm{Cu} 3 \mathrm{O} 7-\delta$ superconducting thin films", Journal of Applied Physics 111, 123911 (2012)

[22] D. E. Oates, S. H. Park, D. Agassi, G. Koren and K. Irgmaier, "Temperature dependence of intermodulation distortion in YBCO: understanding nonlinearity," in IEEE Transactions on Applied Superconductivity, vol. 15, no. 2, pp. 3589-3595, June 2005.

[23] J. Wosik, L. M. Xie, R. Grabovickic, T. Hogan and S. A. Long, "Microwave power handling capability of HTS superconducting thin films: weak links and thermal effects induced limitation," in IEEE Trans. on Applied Superconductivity, vol. 9, no. 2, pp. 2456-2459, June 1999.

[24] L.K.J. Vandamme, M.G. Périchaud, E. Noguera, Y. Danto, U. Behner, “1/f noise as a diagnostic tool to investigate the quality of isotropic conductive adhesive bonds," in IEEE Trans. on components and packaging technology, vol 22, n 3 , sept. 1999, pp. 446-454. 